\title{
La necesidad de problematizar el trabajo infantil. Participación y prepara- ción comunitaria en una localidad socialmente vulnerable de Lima, Perú.
}

\section{The Need of Problematize Child Labor. Community Readiness and Participa- tion in a Socially Vulnerable Locality of Lima, Peru.}

\author{
Anaya, Rogger Holfre y Cueto, Rosa María Luisa Martina \\ Pontificia Universidad Católica del Perú
}

(Rec: abril de 2018 - Acept: noviembre de 2018)

\begin{abstract}
Resumen
El objetivo de estudio fue analizar la relación entre la participación y la preparación comunitaria frente al trabajo infantil, tomando como eje principal el contexto comunitario en una muestra intencionada de 76 mujeres y hombres miembros de una comunidad y 4 informantes clave de la localidad. Se aplicaron encuestas a los miembros de la comunidad, y se realizaron entrevistas semiestructuradas a los informantes clave. Se evaluó la estructura factorial y consistencia interna de las escalas, obteniéndose dimensiones específicas. Se analizó las variables a través de correlaciones y regresión lineal múltiple, encontrando modelos que explican la varianza de participación y preparación comunitaria entre el $15 \%$ al $46 \%$. Las entrevistas situaron a la comunidad en un nivel de "negación/resistencia" al cambio frente al trabajo infantil. Así, los resultados sugieren la necesidad de problematizar esta temática desde la comunidad y las propuestas de intervención, pues si bien existe una participación activa en general, se mantiene una visión ambigua acerca del trabajo infantil, así como una baja disposición a actuar colectivamente frente a esta problemática.
\end{abstract}

Palabras clave: Preparación comunitaria, participación comunitaria, trabajo infantil.

\begin{abstract}
The aim of the study was to analyze the relationship between community readiness and participation concerning child labor taking as the main axis the community context of a purposive sample of 76 men and women community members and 4 local key informants. Surveys were applied on community members and semi-structured interviews were conducted to local key informants. The factorial structure and internal consistency of the scales were evaluated, obtaining specific dimensions. The variables were analyzed by multiple linear regression and correlations, evidencing models that explain the variance of community readiness and participation between $15 \%$ and $46 \%$. The interviews placed the community in a level of "deny/resistance" to change when facing child labor. Thus, the results suggest the need to problematize this subject from the community and intervention proposals, because although there is an active participation in general, an ambiguous view remains on child labor, as well as a low willingness to act collectively on this issue.
\end{abstract}

Key words: Community Readiness, Community Participation, Child Labor

Correspondencia: Dirección de correspondencia: Ma. Anaya, Rogger Holfre. Pontificia Universidad Católica del Perú, Avenida Universitaria 1801, San Miguel, Lima, Lima, Perú. email: anaya.rogger@pucp.pe 
El trabajo infantil es un fenómeno de larga data que viene siendo atendido por países de América Latina y el Caribe firmantes de los convenios $N^{\circ} 138$ y 182 de la Organización Internacional del Trabajo-OIT. En Perú, la normativa provista por el Ministerio de Trabajo y Promoción del Empleo-MTPE regula el trabajo infantil entre los 14 y 18 años estableciendo condiciones mínimas requeridas para la seguridad y el desarrollo de niños y niñas involucrados en estas labores. Además, prohíbe el trabajo infantil en cualquier modalidad entre 5 y 13 años (MTPE, 2012). De acuerdo al Instituto Nacional de Estadística e Informática del Perú-INEI (2018) cerca de dos millones de niñas, niños y adolescentes en Perú trabajan, el 17.5\% tienen 5 a 13 años y el $45 \%$ tienen 14 y 17 años. Al respecto, se ha encontrado que la inserción laboral temprana, sobre todo durante la educación básica, limita las oportunidades de desarrollo y acceso a empleos, calidad de vida y educación superior (Alarcón, 2001). Sin embargo, pese a los esfuerzos para reducir el trabajo infantil no regulado e informal, dentro o fuera del hogar (Alarcón, 2001; Organización Internacional del Trabajo, 2012; Silva, 2010), el porcentaje de niños y niñas involucrados en trabajo infantil en el país sigue siendo alto y se mantiene en prevalencia en relación a años previos (INEI, 2018).

Según el área de residencia, la mayor cantidad de niñas y niños que trabajan (64\%) viven en zonas rurales, y que el $63.5 \%$ de los que trabajan en zonas urbanas son adolescentes entre los 14 y 17 años (INEl, 2015). Si bien existen diferencias en el trabajo infantil, en zonas rurales el trabajo parte por ser un medio de socialización e involucramiento en la vida comunal de niñas y niños que tiene repercusión en la economía familiar (INEI, 2015, Liebel, 2003; Simón, 2015); mientras que, en zonas urbanas, el trabajo infantil se encuentra menos visible y, por ende, más vulnerable (c.f. Cussiánovich, 2009; Simón, 2015). Además, el promedio de horas-trabajo alcanza a ser 10:48, en el caso de niños entre 5 a 13 años y 21:59 horas para adolescentes entre 14 y 17 años (INEl, 2018).

La tradición del trabajo infantil en zonas rurales del Perú, con ciertas transformaciones, se mantiene vigente en las familias que migraron a zonas urbanas, principalmente a Lima, quienes mantienen la concepción del trabajo infantil como espacio de socialización (Cussiánovich, 2009; Liebel, 2003). Organizaciones como el Instituto de Formación de Adolescentes y Niños Trabajadores-INFANT y el Instituto de Formación para Educadores de Jóvenes, Adolescentes y Niños Trabajadores-IFEJANT, ven a las comunidades urbanas como escenarios clave para implementar iniciativas y programas que protejan y brinden actividades seguras a niñas, niños y adolescentes que trabajan, impulsando la educación y el desarrollo de habilidades emprendedoras (Cussiánovich, 2009; Liebel, 2003). Si bien existe evidencia que en algunas zonas de Lima las comunidades han ido asumiendo un rol más activo frente al trabajo infantil (c.f. Bernal, Basto, Sarmiento, \& Rincón, 2011; Taft, 2013), las cifras muestran que la proporción de niñas, niños y adolescentes que trabajan aún son altas (INEI, 2015) y la intensidad de horas en el trabajo son elevadas (INEI, 2018).

La persistencia del trabajo infantil se atribuye a la escasez de recursos económicos de las familias, así como a necesidades básicas insatisfechas en las comunidades (Alarcón, 2001; Bernal et al., 2011). Sin embargo, desde la literatura en psicología en Lima-Perú no se encuentra registro de estudios que analicen o problematicen al trabajo infantil desde las comprensiones y necesidades de las comunidades socialmente vulnerables. Así, la psicología comunitaria podría aportar a entender la complejidad de este fenómeno, al revelar cómo se relacionan las comunidades con el trabajo infantil y promover la participación de los distintos actores sociales involucrados (Montenegro, 2004; Montero, 2003; Serrano \& López, 2008). De acuerdo con los principios de la psicología comunitaria, la problematización es un proceso clave de reflexión crítica para la desnaturalización de problemáticas normalizadas o hegemónicas en los colectivos, que apunta a impulsar el cambio social respecto a condiciones que generan situaciones perjudiciales que son naturalizadas (Berroeta \& Rodríguez, 2010; Fernández, Morales \& Molero, 2011; Martínez, 2006). Así, la disciplina permite analizar, desde las relaciones y la estructura comunitaria, situaciones que vulneran derechos y generan dificultades para el pleno desarrollo de sus miembros (Holgado, Maya-Jariego, Ramos, Palacio, Oviedo-Trespalacios, Romero \& Amar, 2012; Simón, 2015).

Así, el 2013 en Perú, se desarrolla el proyecto de participación-acción llamado "Evaluación de factores psicosociales, educativos y comunitarios relacionados con una propuesta de intervención en la prevención del trabajo infantil en Lima", desarrollado por el Departamento de Psicología y el Departamento de Educación, de la Pontificia Universidad Católica del Perú-PUCP. Este proyecto tuvo carácter interdisciplinario en su diseño e implementación y tomó de referencia la experiencia de "Edúcame primero Colombia" desarrollado por la Universidad de Sevilla y la Universidad del Norte de Colombia (Holgado et al., 2012). Asimismo, contó con la participación voluntaria de estudiantes de pregrado de las facultades de Psicología y Educación para la ejecución de las actividades del proyecto, tanto a nivel de intervención como de investigación.

En el marco del proyecto, la estrategia "Crecer Jugando" se orientó a impulsar el fortalecimiento de habilidades sociales en niñas y niños de 3ero y 4to grado de primaria a través del juego y actividades lúdico-educativas con las que se reforzaba el aprendizaje de las materias impartidas en una escuela. Así, el presente artículo discute los resultados de la evaluación de los niveles de preparación comunitaria y de participación de la comunidad involucrada en el proyecto.

\section{Participación comunitaria}

Se define participación comunitaria como el proceso social que involucra a diferentes agentes de la comunidad, quienes comparten alguna necesidad, y tratan de identificarla para tomar decisiones con la finalidad de generar mejoras (Montero, 2004). El involucramiento de comunidades en iniciativas de intervención mejora la prestación y recepción de los servicios de las mismas y que, por el contrario, programas de desarrollo y de salud fracasarían sin la participación de la comunidad (Fernández, Morales \& Molero, 2011; Musitu, Herrero, Cantera \& Montenegro, 2004). Un efecto psicológico importante es que la acción de las personas apoya su agencia para gestionar cambios en la comunidad (Maya-Jariego, 2004). Diversos estudios reportan que a través de la participación la comunidad identifica recursos propios y del entorno, que ayudan a las personas a desarrollar estrategias para el logro de metas (Serrano-García \& López-Sánchez, 2008; Montenegro, 2004; Montero, 2003), y promueven un mayor sentimiento de control sobre el ambiente (Calderón \& Bustos, 2007; Montero, 2004; Páez \& Espinosa, 2010; Wiesenfeld \& Sánchez, 2001). 
Un aspecto sumamente positivo de la participación comunitaria es que favorece el desarrollo de un mayor sentimiento de involucramiento y promueve la problematización sobre situaciones que pueden encontrarse naturalizadas (Montero, 2004; Troudi, Harneckery \& Bonilla, 2005). Además, el desarrollo de lazos de confianza y de cohesión entre las personas resultaría ser un buen predictor de participación comunitaria (Freire, 2012). Sin embargo, Balbuena (2012) plantea que los lazos afectivos entre los miembros de una comunidad no implica la percepción de interdependencia, la cual es fundamental para motivar el involucramiento en acciones colectivas.

Asimismo, genera la politización de las personas, al impulsarlas a la reflexión crítica y a actuar frente a problemáticas sociales y desnaturalizarlas (Martínez, 2006; Montero, 2003). Así, la participación comunitaria influye en la interacción y la socialización de las personas e inclusive en la percepción que tengan sobre fenómenos sociales que los rodean, generando cambios sobre la estructura social en el tiempo (Martínez, 2006; Ríos y Moreno, 2009). En este contexto, resulta de utilidad para las intervenciones comunitarias conocer la dinámica interna de la comunidad e identificar factores que potenciarían la participación de sus miembros o, por el contrario, esclarecer posibles obstáculos que merman la acción colectiva, sobre todo con temáticas complejas como el trabajo infantil.

\section{Preparación comunitaria}

Asumir que cualquier intervención obtendrá efectos positivos o que será siempre apropiada para la comunidad, podría llevar a fracasar a muchas propuestas si estas no articulan las necesidades del colectivo con los objetivos institucionales de la intervención (Oetting, Donnermeyer, Plested, Edwards, Kelly \& Beauvais, 1995; Martínez, 2006); más aún, mantener mayor distancia entre lo comunitario y lo institucional afecta la dimensión ética de la intervención comunitaria pues las personas terminan siendo entes pasivos que poco o nada se involucraron en tales propuestas (Winkler, Alvear, Olivares \& Pasmanik, 2014). Por ello, resulta importante conocer cuán dispuesta está una comunidad a afrontar cambios y mantenerlos (Musitu \& Buelga en Musitu, et al., 2004). La preparación comunitaria es definida como la disposición y capacidad de una comunidad para hacer frente a los cambios que implica una intervención (Oetting et al., 1995; Edwards, Jumper-Thurman, Plested, Oetting, \& Swanson, 2000). Así, la preparación comunitaria puede describir el contexto ecológico y organizacional donde se realizan intervenciones orientadas al cambio comunitario (Chilenski, Greenberg \& Feinberg, 2007). Feinberg, Greenberg, y Osgood (2004) plantean que la historia de la comunidad, la efectividad del liderazgo local, así como experiencias de participación y empoderamiento, deben tomarse en cuenta al evaluar la preparación comunitaria. Existen estudios que relacionan la preparación comunitaria con el funcionamiento, mantenimiento y efectividad percibida de una intervención comunitaria (Feinberg et al., 2004; Holgado \& Maya-Jariego, 2012), donde a mayor preparación para el cambio, mayor será la capacidad para garantizar el éxito de la implementación de programas específicos (Flaspohler, Duffy, Wandersman, Stillman \& Mara, 2008). A partir de trabajos realizados por diferentes autores (Oetting et al., 1995; Edwards et al., 2000; Chilenski et al., 2007) se han desarrollado diversos modelos de preparación comunitaria. Edwards y colegas (2000) proponen que los niveles de preparación comunitaria para el cambio requieren de una óptica multidimensional, di- señando el Modelo de Niveles de Preparación Comunitaria [Stage model] (Chilenski et al., 2007), que es una herramienta sencilla para dar retroalimentación rápida sobre una comunidad.

El modelo de "Niveles de preparación comunitaria" presenta seis dimensiones: (1) existencia de iniciativas para hacer frente a problemas, (2) conocimiento acerca del problema en específico, (3) conocimiento de iniciativas implementados para enfrentar el problema en específico, (4) liderazgo de líderes formales y no formales, (5) disponibilidad de recursos accesibles, que pueden ser económicos, materiales, humanos o de alguna otra naturaleza y, (6) clima comunitario, referido a aspectos como clima social, sentido de comunidad y/o cohesión social (Edwards et al., 2000).

La multidimensionalidad del concepto (Edwards et al., 2000) permite describir el contexto comunitario bajo nueve niveles para cada dimensión (Holgado \& Maya-Jariego, 2012). Los nueve niveles del modelo conforman una escala que parte de (1) tolerancia a una problemática existente en la comunidad, (2) negación o ninguna conciencia del problema, (3) vaga conciencia de existencia del problema, (4) idea general de existencia del problema, (5) planificación inicial de acciones centradas al problema, (6) inicio de las acciones, (7) institucionalización y estabilización de programas de intervención, (8) expansión de iniciativas estandarizadas en la comunidad, y (9) profesionalización de las iniciativas comunitarias.

Participación y preparación comunitaria han sido vinculadas al desarrollo y éxito de procesos de cambio en colectivos, aunque se requiere mayor evidencia sobre cómo ambos constructos se relacionan empíricamente. Por ello, el presente estudio tiene como objetivo analizar la relación entre participación y preparación comunitaria frente al trabajo infantil, tomando como eje principal el contexto comunitario de una localidad socialmente vulnerable de Lima Metropolitana.

\section{Participantes}

\section{Método}

Son residentes de la ampliación Pachacamac 4ta etapa del distrito de Villa El Salvador-VES, donde se implementó la estrategia "Crecer Jugando". Se aplicó la adaptación de la guía de entrevista de Edwards y colegas (2000) a cuatro informantes clave, quienes conocen la dinámica interna de la comunidad y las problemáticas locales. Estos fueron dos hombres y dos mujeres entre los 41 y 55 años de edad, migrantes de distintas provincias del Perú; representando a diferentes segmentos de la comunidad: un director de colegio, un pastor evangélico, una líder comunal y una madre de familia.

Fueron encuestados 76 miembros de la comunidad, hombres $(43,4 \%)$ y mujeres (56,6\%), entre 20 a 59 años $(M=38,68, D E$ =9,67). El 92,1\% ha migrado de diferentes provincias hacia Lima y durante su residencia, el $55,26 \%$ ha vivido más de la mitad del tiempo en alguna de las comunidades de la zona. El $82,9 \%$ tiene hijos, mientras que solo el $15,8 \%$ son solteros/as o separados/as.

Cabe mencionar, tanto informantes clave como encuestados no necesariamente eran padres o madres de familia, pues el criterio de selección de participantes era que sean miembros de la comunidad con al menos 3 años de residencia ininterrumpida para conocer la perspectiva de la comunidad en su conjunto sobre el trabajo infantil. 


\section{Instrumentos}

Escala de Participación Comunitaria. Se adaptó la "Escala de participación comunitaria en líderes/as comunitarios/as", elaborada por Seminario (2014), modificando la redacción de algunos ítems hacia actividades de miembros no-líderes de la comunidad. El objetivo de la escala es conocer la intención del colectivo por participar para enfrentar problemas que afectan a la comunidad.

Luego de un análisis de reducción de factores con los 30 ítems de la escala original, se obtuvo una escala de 22 ítems que exploran distintos aspectos de la participación de las personas en su comunidad. La escala presenta respuestas tipo Likert del 1 al 4, donde 1 es "Nada", 2 es "Poco", 3 es "Regular" y 4 es "Bastante". El análisis de componentes principales, utilizando rotación Varimax, presentó una clara estructura factorial $(\mathrm{KMO}=0,71)$ de cinco factores con una varianza explicada de $59,7 \%$. Para obtener las dimensiones finales de la escala de Participación Comunitaria se eliminaron los ítems 4, 5, 10, 12, $14,17,26$ y 29 para mejorar la confiabilidad y la consistencia interna. Se obtuvo una estructura de cinco factores (ver Tabla 1).

Tabla 1. Cargas factoriales para Análisis Factorial con rotación Varimax de la escala de Participación Comunitaria

\begin{tabular}{|c|c|c|c|c|c|}
\hline Cuestionario de Participación comunitaria & $\begin{array}{c}\text { Apoyo y } \\
\text { acción } \\
\text { social }\end{array}$ & $\begin{array}{l}\text { Cohesión } \\
\text { social }\end{array}$ & Confianza & $\begin{array}{l}\text { Estima e } \\
\text { influencia }\end{array}$ & Agencia \\
\hline $\begin{array}{l}\text { 1. Colaboro con las organizaciones y asociaciones de mi co- } \\
\text { munidad. }\end{array}$ &, 50 &, 00 & ,35 & 09 & 14 \\
\hline $\begin{array}{l}\text { 6. Cuando mi comunidad tiene un objetivo todos participan } \\
\text { para lograrlo. }\end{array}$ & ,78 &, 11 & ,04 &,- 31 & 14 \\
\hline 7. Mis vecinos aportan al desarrollo de mi comunidad. &, 57 & ,38 & ,20 &,- 25 &, 33 \\
\hline $\begin{array}{l}\text { 13. Los problemas de mi comunidad necesitan del apoyo de } \\
\text { otros que no viven en ella para poder resolverlos. }\end{array}$ & 74 & ,22 &,- 08 &,- 03 &,- 14 \\
\hline $\begin{array}{l}\text { 18. En mi comunidad todos están comprometidos con me- } \\
\text { jorarla. }\end{array}$ & 65 & 16 &,- 16 &, 54 & , 00 \\
\hline $\begin{array}{l}\text { 19. Acudo a otros miembros de mi comunidad para resolver } \\
\text { mis problemas. }\end{array}$ &, 50 & 29 &,- 30 & ,35 & 24 \\
\hline $\begin{array}{l}\text { 23. Los miembros de mi comunidad se organizan para el de- } \\
\text { sarrollo de los proyectos conjuntos que tenemos. }\end{array}$ & ,77 &,- 03 & 09 & ,27 & 02 \\
\hline 24. Participo en las actividades que organiza mi comunidad. &, 55 & ,41 &,- 18 & 14 & ,29 \\
\hline $\begin{array}{l}\text { 27. Cuando hay un problema en mi comunidad, todos coope- } \\
\text { ramos para solucionarlo. }\end{array}$ & 68 & 03 & 31 & 04 & 08 \\
\hline $\begin{array}{l}\text { 8. Las personas en mi comunidad se sienten bien por par- } \\
\text { ticipar. }\end{array}$ & 11 & 61 & 15 & 27 &,- 14 \\
\hline 9. En mi comunidad las personas participan. & 00 & ,76 & ,02 &,- 02 &, 25 \\
\hline $\begin{array}{l}\text { 11. Los miembros de mi comunidad colaboran para lograr los } \\
\text { objetivos que tenemos en común. }\end{array}$ & 13 & ,74 &,- 01 & 01 & ,04 \\
\hline $\begin{array}{l}\text { 22. Participo en la organización de las actividades sociales y } \\
\text { festividades de mi comunidad. }\end{array}$ & 25, & ,46 & ,30 & 22 & 06, \\
\hline 15. Mi comunidad logra cumplir las metas que se propone. & 18 & ,44 & ,64 & 19 & 12 \\
\hline 16. Me siento feliz de apoyar a mi comunidad. & 00 &,- 09 & ,79 & 01 & 14 \\
\hline $\begin{array}{l}\text { 20. Los miembros de mi comunidad se apoyan entre ellos } \\
\text { para resolver sus problemas. }\end{array}$ & 02 & ,30 & ,62 & ,34 &,- 22 \\
\hline 21. Me parece importante los proyectos de mi comunidad. & 09 & ,22 & ,41 &, 54 & 07 \\
\hline $\begin{array}{l}\text { 28. La opinión de todos es escuchada y tomada en cuenta } \\
\text { por los demás miembros de mi comunidad. }\end{array}$ & 11 & 13, & 06, & ,57 & 43, \\
\hline 30. En las asambleas se toman decisiones democráticamente. &,- 06 & 02 & 18 & ,66 & 17 \\
\hline 2. Yo aporto al desarrollo de mi comunidad. & ,36 & ,08 &,- 15 & 15 &, 52 \\
\hline $\begin{array}{l}\text { 3. Participo en las actividades que mi comunidad realiza } \\
\text { para mejorarla. }\end{array}$ &,- 20 & ,17 & 14 & 11 & ,82 \\
\hline $\begin{array}{l}\text { 25. Los vecinos de mi comunidad ponen de su parte a favor } \\
\text { de la comunidad. }\end{array}$ & ,34 &,- 03 & 14, & 18, & 64, \\
\hline
\end{tabular}

Nota. Cargas factoriales de los ítems incluidos en los factores finales están en Negrita. 
La primera dimensión identificada, apoyo y acción social, explicita la necesidad de colaboración con vecinos para alcanzar objetivos de la comunidad y mejorarla. La segunda, cohesión social, manifiesta un sentido de cooperación con vecinos y comunidad al trabajar en conjunto que, a su vez, implica un sentimiento de satisfacción. La tercera, confianza, expresa un sentido de seguridad frente a la expectativa de superación de obstáculos en la comunidad. La cuarta, estima e influencia, se enfoca en sentirse tomado en cuenta e involucrarse en la toma de decisiones de la comunidad como medio para impulsar la participación. Finalmente, la dimensión agencia, expresa el sentido de empoderamiento que enfatiza al mejoramiento de la comunidad como resultado del esfuerzo colectivo de sus miembros. La consistencia interna fue evaluada a través de alfa de Cronbach: Apoyo y acción social $(\alpha=0,86)$, Cohesión social $(\alpha=0,66)$, Confianza $(\alpha=0,70)$, Estima e influencia $(\alpha=0,57)$ y Agencia $(\alpha=0,56)$.
Escala de Preparación Comunitaria. Se adaptaron al contexto peruano los ítems de la "Escala de preparación comunitaria" (Holgado y Maya-Jariego, 2012). Esta escala evalúa el nivel de preparación de miembros de la comunidad para sumarse a alguna intervención sobre una problemática específica. La escala utilizada presenta un rango de respuestas del 1 al 10, donde 1 es "Nada de acuerdo" y 10 es "Totalmente de acuerdo". El análisis factorial exploratorio, usando rotación Oblimin, presentó una clara estructural factorial $(\mathrm{KMO}=0,85)$ y dio como resultado tres factores con una varianza explicada de $89,9 \%$ : Para obtener las dimensiones finales de la escala de Preparación Comunitaria se eliminaron los ítems 1, 7, 12, 15, 16 y 17 por motivos de confiabilidad. Se obtuvo una estructura de tres factores (ver Tabla 2).

Tabla 2. Cargas factoriales para el Análisis Factorial Exploratorio con Rotación Oblimin del Escala de Preparación Comunitaria

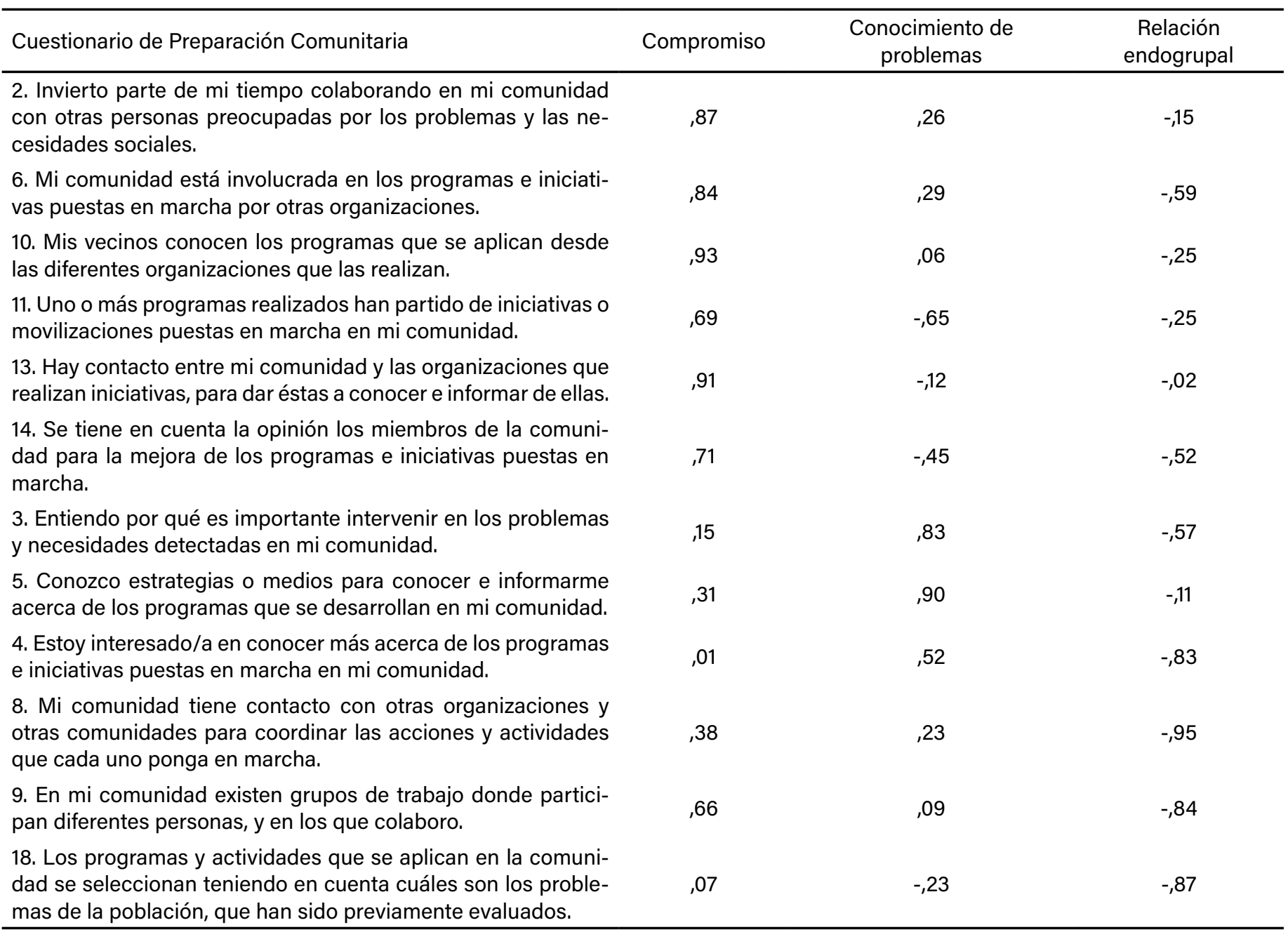

Nota. Cargas factoriales de los ítems incluidos en los factores finales están en Negrita. 
La dimensión, compromiso, expresa la disposición de los miembros de la comunidad para involucrarse e invertir recursos (como tiempo y esfuerzo) en actividades para enfrentar algún problema. Por otra parte, conocimiento de problemas, refiere al manejo de estrategias por parte de la comunidad para informarse y/o comprender una problemática y buscar alguna solución. Finalmente, relación endogrupal, presenta una evaluación subjetiva respecto a lo que uno siente que ha aportado en la comunidad, con la finalidad de decidir involucrarse y participar en actividades/iniciativas o marcar un distanciamiento de ellas, sin que ello implique un desconocimiento del entorno comunitario o una desconexión con los vecinos. La consistencia interna de las dimensiones fue: Compromiso $(\alpha=0,91)$, Conocimiento de problemas $(\alpha=0,85)$ y Relación endogrupal $(\alpha=0,90)$. La escala total obtuvo un Alfa de Cronbach de 0,89 .

Guía de entrevista de Preparación Comunitaria. Se adaptó y tradujo al español la guía de entrevista en profundidad semi-estructurada "Community Readiness Assessment" (Edwards et al., 2000). El instrumento busca conocer la manera en que la comunidad se relaciona con una problemática a través del nivel de preparación en que se ubique. Esta guía cuantifica las seis dimensiones de preparación comunitaria de acuerdo al Modelo de Niveles de Preparación Comunitaria; puntuando del 1 al 9 a la comunidad de acuerdo al nivel alcanzando en cada dimensión y consecutivamente, se promedian los puntajes para cada dimensión de la entrevista. Posteriormente, al obtener las seis puntuaciones totales en cada dimensión, se promedian los puntajes de todas las entrevistas, obteniendo el puntaje total del nivel de preparación comunitario. Las puntuaciones finales son redondeadas hacia abajo, es decir, solo se toma en cuenta los números enteros y no los decimales, para seleccionar el nivel correspondiente.

\section{Procedimiento}

La investigación se centró en una de las zonas de intervención vinculadas a la estrategia "Crecer Jugando". Al tener contacto con el colegio del lugar, se obtuvieron los datos de contacto de padres y madres de familia y el acceso a encuestar a otros miembros de la comunidad, así como datos de posibles informantes clave (entrevistados). La elección de participantes priorizó la facilidad de acceso y la apertura que tenían las personas de participar del estudio. Antes de cada entrevista y encuesta, se usó el consentimiento informado aprobado de acuerdo a los lineamientos del Comité de Ética de la PUCP, en el que se detallaron los objetivos del estudio y se explicitó la condición de voluntariedad y anonimato de participación. Además, en cada parte del levantamiento de información se enfatizó que el objetivo de investigación tenía como tema central al trabajo infantil. Se preguntó por la edad, sexo, estado civil, lugar de nacimiento, tiempo de residencia en Lima y en la comunidad, cantidad de hijos/as, cargo en la comunidad o institución a la que pertenece, y que identificara de manera nominal qué problema identificaba como más importante en su comunidad.

Las entrevistas fueron realizadas en los ambientes donde los informantes desempeñan sus labores. Las encuestas fueron aplicadas casa por casa, invitando a participar a la persona mayor de edad encargada del hogar, que no necesariamente tenía hijos/as pero que sí participaba activamente de la vida comunal y asistía a las asambleas. Se informó a cada participante sobre los objetivos del estudio, tomando en cuenta las consideraciones éticas correspondientes, y se procedió con la resolución de las encuestas.

\section{Análisis de Datos}

Las respuestas de las entrevistas fueron cuantificadas para obtener el nivel de cada dimensión y el nivel total de preparación de la comunidad, siguiendo los protocolos del Modelo de Niveles de Preparación Comunitaria (Edwards et al., 2000). Para ello se elaboró una matriz en el programa Microsoft Excel 2010.

Las encuestas se analizaron empleando el programa de análisis estadístico IBM SPSS20. Se aplicaron pruebas de fiabilidad y consistencia interna a los cuestionarios, identificando factores específicos en cada uno. Se obtuvieron estadísticos descriptivos de la muestra, se realizaron pruebas de normalidad y se analizaron los factores encontrados; asimismo, se realizaron correlaciones $r$ de Pearson y rectas de regresión múltiple para analizar la relación entre las variables.

\section{Resultados}

A continuación, se presentan los resultados obtenidos. En primer lugar, se presentan los resultados descriptivos de ambas variables de interés, para posteriormente presentar los hallazgos de las relaciones encontradas entre ambas a través de los análisis de correlación y de regresión realizados.

\section{Descriptivos de las variables}

Encuestas. A nivel descriptivo, se puede apreciar que tres de las dimensiones de la escala de Participación Comunitaria se encuentran con puntuaciones por encima del punto medio de la escala, cohesión social $(\mathrm{M}=3,01, \mathrm{DE}=$ $0,67)$, confianza $(M=3,11, D E=0,69)$, estima e influencia $(M=3,16, D E=0,62)$ y agencia $(M=2,75, D E=0,69)$; mientras que apoyo y acción social obtiene una puntuación media de 2,55 (DE = 0,74), siendo la más baja.

Respecto a la escala de Preparación Comunitaria, el puntaje total presenta una puntuación ligeramente por encima del punto medio $(M=5,6, D E=1,79)$ expresando tendencia central, al igual que las áreas compromiso $(M=5,72, D E=$ $1,98)$, conocimiento de problemas $(M=5,70, D E=2,73)$ y relación endogrupal $(M=5,43, D E=2,62)$, siendo la más baja y ubicándose por debajo del punto medio.

Entrevistas. El puntaje total de Preparación Comunitaria es 2,54 que corresponde al nivel 2 de "negación/resistencia" al cambio del Modelo de Niveles de Preparación Comunitaria. Asimismo, para las seis dimensiones de preparación comunitaria, las puntuaciones de iniciativas existentes en la comunidad, liderazgo, clima comunitario y recursos corresponden al nivel 2, "negación/resistencia", mientras que conocimiento de las iniciativas y conocimiento sobre el tema se en encuentran en el nivel 3, "vaga conciencia" del problema.

Relación entre Participación y Preparación Comunitaria Los resultados de las correlaciones reflejan relaciones inversas entre las dimensiones de participación y de preparación comunitaria (ver Tabla 3). La dimensión de participación comunitaria apoyo y acción social presenta una correlación alta con el puntaje total de preparación comu- 
nitaria y una correlación medianamente alta con relación endogrupal, y de igual manera presenta correlaciones medianamente bajas con compromiso y conocimiento de problemas. Respecto a las dimensiones de participación comunitaria cohesión social y estima e influencia presentan correlaciones medianamente bajas con relación endogrupal. Finalmente, la dimensión de cohesión social de participación comunitaria correlacionó a un nivel medio bajo con el puntaje total de preparación comunitaria.

Tabla 3. Correlaciones entre Participación y Preparación Comunitaria

\begin{tabular}{|c|c|c|c|c|c|}
\hline Apoyo y acción social & $\begin{array}{c}\text { Cohesión } \\
\text { social }\end{array}$ & Confianza & $\begin{array}{l}\text { Estima e } \\
\text { influencia }\end{array}$ & Agencia & \\
\hline Preparación Comunitaria: Puntaje total &,$- 620 * * *$ &,$- 286^{*}$ &,- 062 &,- 224 &,- 099 \\
\hline Conocimiento de problemas &,$- 293^{*}$ & ,016 & ,194 &,- 028 &,- 129 \\
\hline Relación endogrupal &,$- 561^{* * *}$ &,$- 342 * *$ &,- 203 &,$- 397^{* * *}$ &,- 178 \\
\hline
\end{tabular}

*** $\mathrm{p}<, 001 ;{ }^{* *} \mathrm{p}<, 01 ; * \mathrm{p}<.05$

Consecutivamente, se exploraron las relaciones entre las dimensiones de participación y las de preparación comunitaria aplicando regresión múltiple (ver Figura 1). De esta manera, ubicando a la puntuación de la dimensión de participación comunitaria, apoyo y acción social como variable dependiente y las dimensiones de preparación comunitaria como variables independientes, se obtuvo un modelo significativo $F(3,306)$
$=20,486, p<0,001$, con un $R^{2}=0,46$ de varianza explicada. La dimensión de preparación relación endogrupal, obtuvo un coeficiente multivariado significativo $\beta$ estandarizado $=-0,64$, $\mathrm{p}<0,001,95 \%$ IC $[-0,24,-0,12]$.

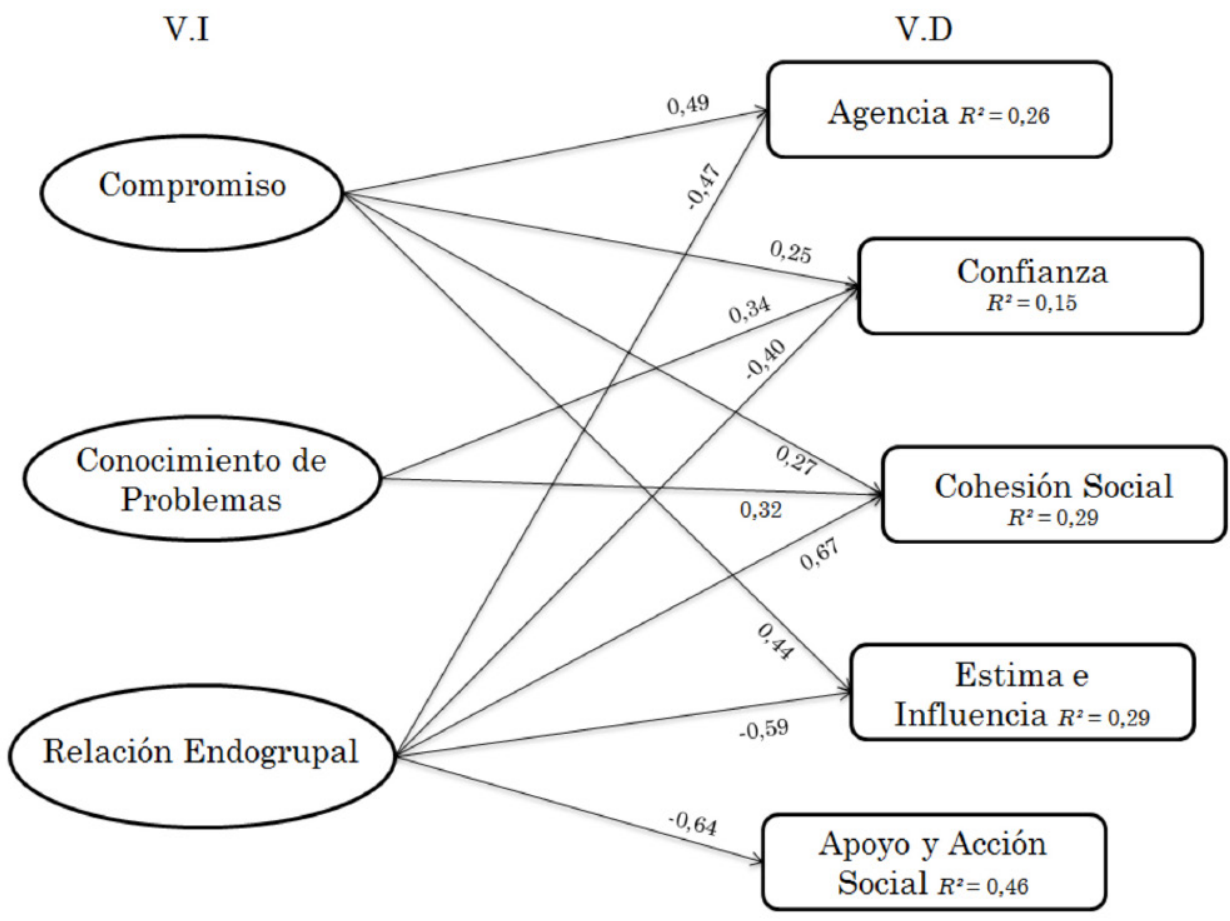

Figura 1. Esquema de regresiones lineales múltiples. Variables independientes (V.I): dimensiones de Preparación Comunitaria. Variables dependientes (V.D): dimensiones de Participación Comunitaria. Varianza explicada por el modelo $\left(R^{2}\right)$.

Figura 1. Esquema de regresiones lineales múltiples. Variables independientes (V.I): dimensiones de Preparación Comunitaria. Variables dependientes (V.D): dimensiones de Participación Comunitaria. Varianza explicada por el modelo $\left(R^{2}\right)$. 
Tomando a cohesión social como variable criterio, se encontró un modelo significativo $F(3,334)=9,953, p<0,001$, con una varianza explicada de $R^{2}=0,54$. Se obtuvieron coeficientes multivariados significativos para las tres dimensiones de preparación comunitaria; relación endogrupal $\beta$ estandarizado $=$ $-0,67, p<0,001,95 \%$ IC $[-0,24,-0,12]$, conocimiento del problema $\beta$ estandarizado $=0,32, p<0,01,95 \%$ IC $[0,02,0,13]$ y compromiso $\beta$ estandarizado $=0,27, p<0,05,95 \%$ IC $[0,02$, $0,17]$. De igual manera, presentando como variable dependiente a la dimensión de participación comunitaria confianza con las dimensiones de preparación comunitaria, se encontró un modelo significativo $F(3,419)=4,339, p<0,01$, con $R^{2}=$ 0,39 de varianza explicada. Los coeficientes multivariados significativos encontrados corresponden a las dimensiones de, compromiso $\beta$ estandarizado $=0,25, p<0,05,95 \%$ IC $[0,01$, $0,17]$, conocimiento del problema $\beta$ estandarizado $=0,34, p<$ $0,01,95 \%$ IC $[0,02,0,15]$ y relación endogrupal $\beta$ estandarizado $=-0,40, p<.01,95 \%$ IC $[-0,18,-0,04]$.

Colocando a la dimensión de participación comunitaria; estima e influencia como variable dependiente y a las dimensiones de preparación comunitaria como variables independientes se obtuvo un modelo significativo $F(3,288)=9,601$, $p<$ 0,001 , con $R^{2}=0,53$. Los coeficientes multivariados obtenidos fueron compromiso $\beta$ estandarizado $=0,44, p<0,001,95 \%$ IC $[0,07,0,21]$ y relación endogrupal ( $\beta$ estandarizado $=-0,59, p<$ $0,001,95 \%[-0.20,-0,08])$. Asimismo, ubicando a la dimensión de participación comunitaria agencia como variable dependiente y las dimensiones de preparación comunitaria como variables independientes, se obtuvo un modelo significativo $F(3,371)=8,362, p<0,001$, con un $R^{2}=0,51$. Se obtuvieron coeficientes multivariados para compromiso $\beta$ estandarizado $=0,49, p<0,001,95 \%$ IC $[0,01,0,25]$ y relación endogrupal ßestandarizado $=-0,47, \mathrm{p}<0,001,95 \%$ IC $[-0,18,-0,05]$.

\section{Discusión}

\section{Participación y preparación comunitaria para el cambio}

Se esperaba encontrar una relación positiva entre ambos constructos, debido a la relación teórica que presentan, pues ambos hacen referencia a movilización y transformación social (Edwards et al., 2000, Holgado \& Maya-Jariego, 2012, Montenegro, 2004, Montero, 2004, Plested, Edwards \& Jumper-Thurman, 2006). Sin embargo, la relación resultó inversa, lo que llevaría a pensar que se emplearon medidas que exploraran diferentes aspectos del fenómeno. Así, es importante resaltar que la escala de participación comunitaria se enfoca en la experiencia de involucramiento y vínculo entre miembros de una comunidad, mientras que la escala de preparación comunitaria se centra en la acción frente a iniciativas concretas de intervención, ya sean promovidas por la comunidad o por un actor externo a ella.

Si bien las medias se consideran altas para el caso de participación comunitaria, ello no implica una disposición comunitaria a involucrarse en nuevas iniciativas colectivas, ya que estas no necesariamente despertarían interés (Balbuena, 2012; Musitu et al., 2004). Se podría interpretar que lo hallado refleja que los participantes percibirían que ya existe un alto sentido de colaboración entre ellos -cohesión social- y satisfacción al respecto, con lo que se sentirían conformes con los resultados de diversas experiencias de participación a lo largo del tiempo. Al respecto, algunos estudios en espacios vulnerables en Lima reportan que la participación comunitaria es más va- lorada, principalmente, en momentos donde las personas deben resolver colectivamente necesidades de infraestructura o gestiones ante las autoridades, así como situaciones donde alguna familia requiere un apoyo concreto, generalmente de tipo simbólico y moral (p.e. entierros, enfermedades, etc.) (Autor 2, 2015; Seminario, 2014; Simón, 2015).

Por otra parte, los resultados obtenidos pueden ser indicio de cierto desgaste del grupo y sus recursos, lo que podría significar que algunos participantes ya no estarían tan dispuestos a participar como podrían haberlo hecho antes (Berroeta \& Rodríguez, 2010; Bronfman \& Gleizer, 1994; Feinberg et al., 2004). Al respecto, se señala que los recursos comunitarios en contextos vulnerables suelen ser limitados y luego de procesos demandantes se debilitarían aún más; la participación comunitaria podría no ser sostenible en estos contextos y más bien, generaría un desgaste a las escasas redes de apoyo de algunas comunidades (Maya-Jariego, 2004).

En la comunidad del estudio, ya se habían gestionado colectivamente los servicios básicos poco tiempo atrás. Así, habría una alta conciencia sobre la efectividad de la acción colectiva -confianza- y una sensación compartida de involucramiento (estima e influencia) en la toma de decisiones (Montero, 2004, Ríos \& Moreno, 2009), lo cual habría permitido el logro anterior. El bajo puntaje reportado en la dimensión de apoyo y acción social, significaría que hoy en día no se percibiría como una necesidad el incremento de acciones comunitarias, en la medida que no se perciben problemáticas para abordar colectivamente.

La relación endogrupal de preparación comunitaria, evidenciaría que los participantes perciben que hacen suficiente por su comunidad, lo cual estaría asociado a la relación inversa obtenida con la dimensión apoyo y acción social de participación comunitaria. Por otro lado, la relación entre relación endogrupal con cohesión social y estima e influencia, sugeriría que el distanciamiento de las actividades comunales, a la larga, incrementaría el sentimiento de exclusión de la toma de decisiones colectivas y, en ese contexto, dejaría de ser relevante la satisfacción obtenida de cooperar con otros (Calderón y Bustos, 2007; Ríos y Moreno, 2009; Wiesenfeld y Sánchez, 2001).

Las relaciones entre apoyo y acción social con compromiso y conocimiento de problemas nos dan a entender que, si bien los participantes no percibirían la necesidad de colaborar con otros en el momento, sí aceptarían eventualmente involucrarse en actividades para hacer frente a problemas. Lo anterior sugeriría que se puede ser consciente de la existencia de necesidades vecinales o comunales, y aun así no estar dispuesto a invertir recursos para informarse o participar de actividades. Ello no significa necesariamente una actitud indiferente frente a los problemas comunitarios, pero sí cierto nivel de tolerancia con una situación problemática, como trabajo infantil (Troudi, Harneckery \& Bonilla, 2005, Wiesenfeld \& Sánchez, 2001).

Asimismo, la teoría señala que no necesariamente una comunidad asimilará como propia una propuesta externa que no considere aspectos ecológicos de acción situada (Martínez, 2006). Concretamente, los resultados sugieren que los participantes estarían dispuestos a sumarse a iniciativas para mejorarla, incluyendo iniciativas para prevenir el trabajo infantil, pero al parecer no se han sentido involucrados en las propuestas gestionadas por actores hoy presentes en la zona. 
Por ello, los espacios de intervención deben propiciar el encuentro entre los objetivos institucionales y las necesidades y dinámicas comunitarias propias (Martínez, 2006; Montero, 2003). En coherencia con ello, la regresión entre apoyo y acción social con relación endogrupal sugiere que a menor percepción de la necesidad de colaboración para lograr objetivos en la comunidad, mayor será el distanciamiento de las acciones colectivas.

Del mismo modo, se encontró que a mayor cohesión social, mayor será el compromiso, pero menor la valoración de la participación en la comunidad -relación endogrupal-. A su vez, la confianza aumentaría dependiendo del incremento del compromiso y el conocimiento del problema, pero sería necesario que disminuyese la evaluación negativa de la acción de participar -relación endogrupal-. Finalmente, para que aumente la estima e influencia y la agencia, será necesario que el compromiso aumente y que disminuya la valoración negativa de la relación endogrupal.

Estos resultados estarían evidenciando la pertinencia de analizar los aspectos subyacentes a las acciones comunitarias de modo integrado, considerando el rol tanto de aspectos vinculados con las relaciones interpersonales (Freire, 2012), de las valoraciones que los miembros de la comunidad otorguen a la participación como medio para lograr atender sus necesidades (Autor 2, 2015), así como de la percepción y evaluación de ciertas situaciones de vida como aspectos problemáticos o realidades que deben transformarse (Montenegro, 2004). Así, el conocimiento de las dinámicas psicosociales y comunitarias vinculadas con las problemáticas sociales específicas (p.e. actitudes, conocimiento, prácticas), y la memoria compartida sobre experiencias de acción colectiva pasadas (Balbuena, 2012) resultan ser de suma relevancia en el desarrollo de iniciativas de trabajo comunitario (Maya-Jariego, 2004), pues estarían a la base de la disposición y el interés de la comunidad por involucrarse activamente en la solución de problemas necesarios de enfrentar colectivamente.

\section{Preparación comunitaria frente al trabajo infantil}

Queda claro que el trabajo infantil es una problemática necesaria de abordar, principalmente en contextos de alta vulnerabilidad social (Alarcón, 2001; Liebel, 2003; Simón, 2015). La información recabada nos señala que en efecto, existe el compromiso de una serie de instituciones que invierten recursos, específicamente en la comunidad del estudio, con miras a prevenir y mitigar el trabajo infantil (Cussiánovich, 2009; Taft, 2013). Sin embargo, los resultados encontrados nos presentan una incoherencia entre el esfuerzo de los actores externos y la percepción y motivación de los miembros de la comunidad por actuar frente a esta situación. Al respecto, la acción colectiva frente al trabajo infantil se había limitado a la recepción de útiles escolares entregados por algunas organizaciones privadas, y la asistencia de algunos niños y niñas a sesiones de reforzamiento escolar por las tardes, según expresaron los informantes clave.

Los resultados obtenidos nos indican que frente al trabajo infantil, la comunidad se encuentra en un nivel dos de preparación comunitaria, esto es de "negación/resistencia" al cambio. Se podría interpretar que los participantes se encontrarían enterados de programas o proyectos existentes en la zona, pero que no estarían involucrándose en ellos (Martínez, 2006). Contrastando esta información con los datos de la encuesta y con estudios previos en la zona, se evidencia que se mantiene una visión ambigua sobre el trabajo infantil y la necesidad de regularlo o eliminarlo (Simón, 2015). Entonces, ante la falta de problematización clara acerca del trabajo infantil y sus consecuencias, la comunidad no realizará esfuerzos propios para hacerle frente, lo que termina por invisibilizar la realidad del trabajo infantil al interior de la comunidad (Bronfman \& Gleizer, 1994; Fernández, Morales \& Molero, 2011).

Uno de los hallazgos más relevantes del estudio sería que el trabajo infantil no estaría siendo considerado una problemática que requiera de la toma de acción de la comunidad. Por ello, la falta de motivación por involucrarse en acciones concretas se derivaría de la negación de la existencia de un problema por parte de la comunidad (Holgado \& Maya-Jariego, 2012). El trabajo infantil es un problema para los actores externos que actúan en la comunidad, no así para los miembros de la misma (c.f. Simón, 2015), a pesar que estos últimos demostraron capacidad y efectividad en acciones colectivas en el pasado, cuando estas emergieron genuinamente de una necesidad sentida por la comunidad (Montero, 2004) como lo fue la instalación de servicios básicos, que gestionaron colectivamente.

Por otra parte, es posible que la influencia de las tradiciones y prácticas culturales de los miembros de la comunidad, en su mayoría provenientes de las zonas rurales del Perú, mantengan la concepción del trabajo como parte del proceso adaptativo de integración de las niñas y niños en sus comunidades, y configuración de su mundo social (Cussiánovich, 2009; Simón, 2015). Esta mirada alternativa respecto al trabajo infantil debería ser un elemento que integre en el proceso de problematización a la comunidad y la academia, que permita iniciar procesos de desnaturalización del fenómeno, de involucramiento de la comunidad y de profesionales afines en la búsqueda de alternativas para la prevención y mitigación de formas de trabajo que explotan y vulneran a niñas y niños (Martínez, 2006). Ello favorecería el uso eficiente de recursos que la comunidad ha desplegado en procesos previos de movilización y participación comunitaria, y permitiría generar otras alternativas para comprender este fenómeno en Latinoamérica.

\section{Conclusiones}

Los resultados evidencian que participación y preparación comunitaria se encuentran asociadas. También, se hace patente el rol que tendrían otros factores, como el vínculo comunitario y la evaluación sobre los logros alcanzados colectivamente ante la disposición al cambio y el involucramiento en iniciativas comunitarias.

Respecto a los objetivos del estudio, la metodología utilizada (combinando entrevistas a informantes clave con encuestas e integrando los resultados) permitió obtener información relevante acerca de los alcances y significados de las variables estudiadas, asegurando de esta manera, la validez ecológica de las conclusiones del estudio, de suma importancia para obtener resultados con calidad y pertinencia local (Montero, 2003).

De igual manera, queda señalada la pertinencia y utilidad de evaluar los niveles de participación y de preparación comunitaria en el marco de las intervenciones comunitarias, dada la vinculación entre ambas variables, así como su rol fundamen- 
tal en la movilización de la comunidad. Además, se identifican dos desafíos para el estudio de fenómenos sociales de interés para los psicólogos comunitarios. En primer lugar, el estudio demuestra la importancia de contar con herramientas técnicas adecuadas de obtención de información para comprender las dinámicas psicosociales y colectivas que son sustento de los procesos de cambio comunitario. Por otro lado, se confirma la necesidad de fomentar procesos de problematización en el contexto de diálogo entre academia y comunidad (Martínez, 2006; Silva, 2010), así como entre comunidad, instituciones estatales y organismos privados que implementan acciones. Esto pues, al asegurar una mayor aproximación entre lo institucional y las necesidades de la comunidad, se genera mayor sostenibilidad y las personas se apropian de la intervención, favoreciendo el protagonismo de la comunidad y el fortalecimiento de sus recursos en estos procesos.

\section{Referencias}

Alarcón, W. (2001). ¿Por qué erradicar el trabajo infantil? Conferencia en el Seminario-Taller Nacional organizado por la Red por un Futuro sin Trabajo Infantil, 1819 de setiembre. Extraído de http://www.oocities. org/gin_peru/alarcon-trabajoinf.htm

Balbuena, A. (2012). Sentido de comunidad, bienestar y memoria colectiva en una comunidad rural de la costa norte peruana. Tesis de licenciatura. Lima: Pontificia Universidad Católica del Perú.

Bernal, A., Basto, Y., Sarmiento, A., \& Rincón, S. (2011). Educación y Trabajo: Un encuentro de experiencias y saberes colectivos. Revista Internacional desde los Niños/as Adolescentes Trabajadores, 20, 71-76.

Berroeta, H., \& Rodríguez, M. (2010). Una Experiencia de Participación Comunitaria de Regeneración del Espacio Público. Revista Electrónica de Psicología Política, 8(22), 1- 26. Extraído de http://www.psicopol. unsl.edu.ar/abril2010_Nota1.pdf

Bronfman, M., \& Gleizer, M. (1994). Community participation: need, excuse, or strategy? What are we talking about when we refer to Community Participation? Cad. Saúde Pública, 10 (1), 111-122. doi: 10.1590/ S0102-311X1994000100012

Chilenski, S., Greenberg, M., \& Feinberg, M. (2007). Community readiness as a multidimensional construct. Journal of Community Psychology, 35(3), 347-365. Autor 2. (2015).

Cussiánovich, A. (2009). Ensayo sobre Infancia II. IFEJANT. Lima.

Edwards, R., Jumper-Thurman, P., Plested, P., Oetting, E. \& Swanson, L. (2000). Community Readiness: research to practice. Journal of Commmunity Psychology, 28(3), 291-307.

Feinberg, M., Greenberg, M., \& Osgood, D. (2004). Readiness, Functioning, and Perceived Effectiveness in Community Prevention Coalitions: A Study of Communities That Care. American Journal of Community Psychology, 33(3-4), 163-176.

Fernández, I., Morales, F., \& Molero, A. (2011). Psicología de la Intervención Comunitaria. Bilbao: Desclée de Brouwer.

Flaspohler, P, Duffy, J., Wandersman, A., Stillman, L., \& Maras, M. (2008). Unpacking Prevention Capacity: An Intersection of Research-to-practice Models and Community-centred Models. American Journal of
Community Psychology, 41(3-4), 182-196.

Freire, S. (2012). Identificación con el lugar, participación y clima emocional en una comunidad rural de la costa norte del Perú. Tesis de Licenciatura. Lima: Pontifica Universidad Católica del Perú.

Holgado, D., \& Maya-Jariego, I. (2012). Preparación comunitaria y contextos de intervención: el caso de los trabajadores sociales de atención primaria en Andalucía (España). Anales de Psicología, 28(1), 150-160.

Holgado, D., Maya-Jariego, I., Ramos, I., Palacio, J., Oviedo-Trespalacios, O., Romero, V., \& Amar, J. (2012). Impact of Child Labor on Academic Performance: Evidence from the Program "Edúcame Primero Colombia". International Journal of Educational Development, http://dx.doi.org/10.1016/j.ijedudev.2012.08.004

Instituto Nacional de Estadística e Informática (2018). Perú: Línea de base de los principales indicadores disponibles de los Objetivos de Desarrollo Sostenible (ODS). Lima, Perú: Instituto Nacional de Estadística e Informática. Extraído de https://www.inei. gob.pe/media/MenuRecursivo/publicaciones_digitales/Est/Lib1578/libro.pdf

Instituto Nacional de Estadística e Informática (2015). Perú: Perfil del Trabajo Infantil al 2013. Lima, Perú: Instituto Nacional de Estadística e Informática. Extraído de https://www.inei.gob.pe/media/MenuRecursivo/publicaciones_digitales/Est/Lib1207/ Libro.pdf

Liebel, M. (2003). Infancia y Trabajo. Lima: Ifejant.

Martínez, V. (2006). El enfoque comunitario. Estudio de sus modelos de base. Santiago de Chile: Universidad de Chile.

Maya-Jariego, I. (2004). Sentido de comunidad y potenciación comunitaria. Sevilla: Universidad de Sevilla.

Ministerio de Trabajo y Promoción del Empleo (2012). Estrategia Nacional para la Prevención y Erradicación del Trabajo Infantil en el Perú. Lima, Perú: Villena, J., MTPE. Extraído de http://www.trabajo.gob.pe/ archivos/file/exposicion/Estrategia_Trabajo_Infantil.pdf

Montenegro, M. (2004). La investigación acción participativa. En Musitu, G., Herrero, J., Cantera, L. \& Montenegro, M. (Eds.). Introducción a la Psicología Comunitaria (pp. 79-87). Barcelona: Editorial UCO.

Montero, M. (2003). Teoría y práctica de la Psicología Comunitaria. La tensión entre comunidad y sociedad (3era ed.). Buenos Aires: Editorial Paidós.

Montero, M. (2004). Introducción a la psicología comunitaria. Desarrollo, conceptos y procesos. Buenos Aires: Editorial Paidós.

Musitu, G., Herrero, J., Cantera, L., \& Montenegro, M. (2004). Introducción a la Psicología Comunitaria. Barcelona: UCO.

Oetting E., Donnermeyer J., Plested B., Edwards R., Kelly, K., \& Beauvais F. (1995). Assessing community readiness for prevention. The International Journal of the Addictions, 30(6), 659-683.

OIT (2012). Informe mundial sobre el trabajo infantil 2012. Vulnerabilidad económica, protección social y trabajo infantil. Ginebra: OIT.

Plested, B., Edwards, R., \& Jumper-Thurman, P. (2006). Community Readiness: A Handbook for a Successful Change. Fort Collins, CO: Tri-Ethnic Center for 
Prevention Research.

Ríos, L., \& Moreno, P. (2009). Influencia de la participación comunitaria y la identidad con el lugar en la satisfacción vital en inmigrantes. Escritos de Psicología, 3(2), 8-16. Extraído de http://scielo.isciii.es/scielo. php?pid=S1989- 38092010000100002\&script=sci_arttext

Seminario, M. (2014). Sentido de comunidad, participación comunitaria y valores en lideres/as comunitarios/ as en contextos de vulnerabilidad social. Tesis de licenciatura. Lima: Pontificia Universidad Católica del Perú.

Serrano-García, I., \& López-Sánchez, G. (2008). Dos décadas de desarrollo de la psicología social comunitaria: De Puerto Rico al mundo. San Juan: Publicaciones Puertorriqueñas.

Silva, G. (2010). Voces sobre el trabajo infantil. Actitudes y vivencias de padres, madres y maestros de niños que trabajan. Lima: IEP.

Simón, A. (2015). Creencias y Actitudes sobre la Infancia y Trabajo Infantil en su Relación con los Valores en un Contexto de Vulnerabilidad. Tesis de Licenciatura. Lima: Pontificia Universidad Católica del Perú.

Taft, J. (ed.) (2013). Nothing About Us, Without Us: Critiques of the International Labor Organization's Approach to Child Labor from the Movements of Working Children. [Número especial]. Instituto de Formación para Educadores de Jóvenes, Adolescentes y Niños Trabjadores de América Latina y el Caribe. Lima: Perú

Troudi, E., Harnecker, M., \& Bonilla, L. (2005). Herramientas para la participación. Caracas: Editorial Servi-K.

Winkler, M. I., Alvear, K., Olivares, B., \& Pasmanik, D. (2014). Psicología Comunitaria hoy: Orientaciones éticas para la acción. Psicoperspectivas, 13(2), 44-54. Extraído de https://scielo.conicyt.cl/pdf/psicop/ v13n2/art05.pdf

Wiesenfeld, E., \& Sánchez, E. (2001). Sustained participation: A community based approach to adressing environmental problems. En: R. Bechtel \& A. Churchman (Eds.), Handbook of Environmental Psychology (pp. 629-643). New York: John Wiley\&Sons. 